\title{
CONSISTENCY AND SYMMETRY CONDITIONS FOR PLANE PROJECTIONS
}

\author{
G. KonTRYM-SZNAJd \\ W. Trzebiatowski Institute of Low Temperature and Structure Research \\ Polish Academy of Sciences, P.O. Box 937, 50-950 Wrocław 2, Poland
}

Interdependences between plane projections of densities $\rho(p)$ for various crystallographical structures are derived from the conditions of both the consistency and symmetry of projections. Some additional relations are obtained by treating plane projections as line projections of $\tilde{\rho}_{\mathrm{L}}(p)$ ( $\tilde{\rho}_{\mathrm{L}}$ is a line projection of $\rho(p)$ ) and using the consistency and symmetry conditions for the line projections. The relations found can be utilized for both an improvement of experimental spectra and a verification of various techniques used for e.g. correcting Compton profiles.

PACS numbers: 71.20.-b, 78.70.Bj

\section{Introduction}

All experimental spectra, being projections of the same function $\rho(p)$, must be interdependent. This dependence can be derived from both the consistency and symmetry conditions, presented by us for line projections in the paper [1]. Here we discuss this question for plane projections, as measured e.g. in the Compton scattering experiment

$$
J\left(p_{z}\right)=\int_{-\infty}^{\infty} \int_{-\infty}^{\infty} \rho(p) \mathrm{d} p_{x} \mathrm{~d} p_{y}
$$

where $\rho(p)$ is the electron density in the extended momentum space $p$.

Due to the symmetry of $\rho(p), J\left(p_{z}\right)$ can be expanded into lattice harmonics

$$
J\left(p_{z}\right) \equiv J_{\beta, \alpha}(p)=\sum_{l \nu} g_{l \nu}(p) F_{l \nu}(\beta, \alpha),
$$

where $F_{l \nu}$ form an orthogonal set of linear combinations of spherical harmonics $Y_{l}^{m}$ of the order $l$. Index $\nu$ distinguishes harmonics of the same order and $(\beta, \alpha)$ describe the azimuthal and the polar angles of the $p_{z}$-axis with respect to the reciprocal lattice system and $p=\left|p_{z}\right|$.

Equation (1) can be solved analytically if the radial functions $g_{l \nu}$ are expanded into Hermite [2] or Jacobi [3] polynomials

$$
g_{l \nu}(p)=\sum_{m=0} a_{l m} \mathrm{e}^{-p^{2}} H_{l+2 m}(p),
$$




$$
g_{l \nu}(p)=\sum_{m=0}^{\infty} b_{l m}\left(1-p^{2}\right) P_{l+2 m}^{(1,1)}(p)
$$

which is equivalent to the fact that the lowest polynomial in $g_{l \nu}$ is of the order $l$. This property allows to estimate some dependences between $J\left(p_{z}\right)$ given in this paper.

\section{Consistency and symmetry conditions}

The consistency condition (CC) for $J\left(p_{z}\right)$ is always satisfied if the measured spectra are complete (for more details see [1]) which is usually true in the Compton profiles (CP) experiment. This condition is automatically imposed on the data via the reconstruction of $\rho(p)$ but it could be also utilized for checking if our data were measured and corrected properly.

In order to check if spectra are proper the following procedure is proposed:

1. Functions $g_{l \nu}(p)$ are evaluated from Eq. (2).

2. Each of $g_{l \nu}(p)$ is expanded into a series of any even orthogonal polynomials $\sum_{m=0} \tilde{a}_{l m} W_{2 m}(p)^{*}$.

3. We check if the first $l / 2$ coefficients $\tilde{a}_{l}^{m}$ are close to zero in comparison with the first $l / 2$ coefficients $\tilde{a}_{0}^{m}$ (see discussion in Sec. 3 ). If not, there is some inconsistent error in the data which is bigger than the statistical noise and our data should be proved once more. If this ratio is small, we can correct $J\left(p_{z}\right)$ putting $l / 2$ first $\tilde{a}_{l}^{m}$ equal to zero and using Eq. (2) with the consistent functions $g_{l \nu}(p)$ which fulfil the CC following from Eqs. (3) or (4).

However, sometimes we are not able to calculate $g_{l \nu}(p)$ - if e.g. for the hcp structure we have only three $J\left(p_{z}\right)$ with $p_{z}$ along the $\Gamma M, \Gamma K$ and $\Gamma A$ symmetry directions. In this case $(\beta, \alpha)$ are equal to: $(\pi / 2, \pi / 6),(\pi / 2,0)$ and $(0,0)$, respectively, while the first three lattice harmonics are independent of $\alpha$ (have the same values for the $\Gamma M$ and $\Gamma K$ directions). Therefore, here we propose to use quite different analysis, treating plane projections of $\rho(p)$ as line projections of $\tilde{\rho}_{L}$ (defined below) and using the consistency and symmetry conditions for line projections [1].

Let us treat $J\left(p_{z}\right)$ as a line projection of $\tilde{\rho}\left(p_{z}, p_{y}\right)$ along $p_{y}$

$$
J\left(p_{z}\right)=\int_{-\infty}^{\infty} \tilde{\rho}\left(p_{z}, p_{y}\right) \mathrm{d} p_{y},
$$

with $\tilde{\rho}$ being a line integral of $\rho(\boldsymbol{p})$ along some particular direction $p_{x}$, i.e.

$$
\tilde{\rho}\left(p_{z}, p_{y}\right) \equiv J\left(p_{z}, p_{y}\right)=\int_{-\infty}^{\infty} \rho(\boldsymbol{p}) \mathrm{d} p_{x} .
$$

Choosing $p_{x}$ along an axis of the crystal rotation of the order $|G|$, all spectra $J\left(p_{z}\right)$ (with $p_{z}$ changed on the plane perpendicular to this fixed $p_{x}$ axis) can be expanded into the following series:

$$
J\left(p_{z}\right) \equiv J(p, \varphi)=\sum_{n=0}^{\infty} J_{n}(p) \cos (n \varphi),
$$

*'This has an additional advantage - having the least-squares approximation properties, we take properly experimental errors into account. In such a case when we do not perform reconstruction, we propose to use Chebyshev polynomials for the reasons described in the paper [1]. 
where $n=0 \bmod |G|$. Now $p_{z} \equiv(p, \varphi)$ is described in the polar system with $p=\left|p_{z}\right|$. This means that all nonequivalent directions $p_{z}$ are given by $\varphi \in\left(0, \varphi_{G}=\pi /|G|\right)$ and $J\left(p_{z}\right)$ is a periodic function with respect to $\varphi$.

Let $c_{m}(\varphi)$ denote coefficients of the expansion $J(p, \varphi)$ into some orthogonal polynomial series. Knowing both the consistency and the symmetry conditions for line projections [1], the following dependence between $c_{m}(\varphi)$ occurs:

$1^{\circ} .|G| / 2$ first $c_{m}(\varphi)$ is the same $\left(c_{m}(\varphi)=c_{m}\right)$,

$2^{\circ}$. $|G|$ first coefficients $c_{m}(\varphi)$ satisfy a dependence: $2 c_{m}\left(\varphi_{G} / 2\right)=c_{m}(\varphi)+$ $c_{m}\left(\varphi_{G}-\varphi\right)$, for all $J\left(p_{z}\right)$ with $p_{z}$ lying on the plane perpendicular to the rotation axis of the order $|G|$. Therefore, for our three spectra $J\left(p_{z}\right)$ (denoted here as $J(\Gamma M), J(\Gamma K)$ and $J(\Gamma A))$ we will get that $c_{i}(\Gamma M)=c_{i}(\Gamma K)$ for $i=0$, $1,2$ (here $|G|=6)$ and $c_{0}(\Gamma A)=c_{0}(\Gamma M)=c_{0}(\Gamma K)(|G|=2)$ where $c_{0}$ denotes the norm of the spectra. This allows to check if two spectra $(J(\Gamma M)$ and $J(\Gamma K))$ are consistent. In order to check $J(\Gamma A)$ we should have at least one additional spectrum $N(\Gamma I)$ (for $\beta=\pi / 4$ and e.g. $\alpha=0$ ), i.e. with $p_{z}$ on the plane with symmetry $|G|=2$ where the coefficient $c_{1}$ has a property: $2 c_{1}(\Gamma I)=c_{1}(\Gamma A)+c_{1}(\Gamma K)$. This equality is satisfied for $\beta=\pi / 4$ and any $\alpha=\alpha_{k} \in(0, \pi / 6)$, i.e. $2 c_{1}(\Gamma I)=c_{1}(\Gamma A)+c_{1}(\Gamma N)$. Here the $\Gamma N$ and the $\Gamma I$ directions are defined for $\left(\beta=\pi / 2, \alpha_{k}\right)$ and $\left(\beta=\pi / 4, \alpha_{k}\right)$, respectively. Knowing that $c_{1}(\Gamma N)=c_{1}$ we obtain that $c_{1}(\pi / 4)$ has the same value for each of the spectrum $J\left(p_{z}\right)$ with $p_{z}$ described by $\beta=\pi / 4$ and arbitrary $\alpha$. It was derived by treating $N(\Gamma M)$ simultaneously as the line projection of $\tilde{\rho}_{\mathrm{L} 1}$ and $\tilde{\rho}_{\mathrm{L} 2}$ with the symmetry $|G|=6$ and $|G|=2$, respectively. $\tilde{\rho}_{\mathrm{L} 1}$ represents the line projection of $\rho(p)$ along the main axis of the rotation while $\tilde{\rho}_{L 2}$ along any line perpendicular to this axis. This last dependence $c_{1}(\beta=\pi / 4, \alpha)=c_{1}$ is particularly interesting because it gives the interdependence between the line projections of different densities $\tilde{\rho}$ while all consistency conditions for line projections are for the same $\tilde{\rho}$. Moreover, it cannot be derived from Eq. (2) because for such projections where $(\beta, \alpha)=(0,0),(\pi / 4,0),(\pi / 2,0),(\pi / 4, \pi / 6),(\pi / 2, \pi / 6)$ the set of equations for $g_{l \nu}$ (Eq. (2)) does not have any solution.

All conditions drawn here for the hcp structure are satisfied for both cubic and tetragonal structures by replacing $|G|=6$ into $|G|=4$. However, for the cubic structures, where three axis of the fourth order exist, one can get some additional rules. Because here directions $\Gamma A(\beta=0, \alpha=0)$ and $\Gamma K(\beta=\pi / 2, \alpha=0)$ are equivalent, we obtain that $c_{1}$ is the same for all projections with $p_{z}$ lying on the main crystallographical planes (independently if they are of the second or fourth order). Some of these results can be derived from Eq. (2) because for the cubic structures all lattice harmonics (except of $F_{0}=1$ ) depend on $(\beta, \alpha)$. Taking the directions [100], [110] and [111] as an example we obtain the following relations for $c_{i}$ :

$$
\begin{aligned}
& 35 c_{i}([100])-8 c_{i}([110])-27 c_{i}([111])=0 \quad \text { for } \quad i=0,1, \\
& c_{i}([100])-4 c_{i}([110])+3 c_{i}([111])=0 \quad \text { for } \quad i=0,1,2 .
\end{aligned}
$$

$c_{1}$ satisfies both equations when $c_{1}([100])=c_{1}([110])=c_{1}([111])$ which is in agreement with the previous result. 


\section{Applications}

Relations $1^{\circ}$ and $2^{\circ}$ for the line projections have been proved for various strongly anisotropic models [1]. Next, having experimental projections for four metals of the hcp structure [4] with $p_{z}$ (plane projections) or $p_{x}$ (line projections) changed on the hcp plane, we checked that the conditions $1^{\circ}$ and $2^{\circ}$ are satisfied with the accuracy of the order $0.5 \%$ up to $1.5 \%$ and $2 \%$ up to $5 \%$, respectively, depending on values of $c_{m}(\varphi)$. Three first $c_{m}(\varphi)$ have the highest values, so here is the lowest influence of the statistical noise and they are best determined.

In order to prove how these conditions will characterize an improper shape of $J\left(p_{z}\right)$, the following situation has been simulated. Let us assume that for some reasons one projection is given incorrectly, e.g. it is measured for somewhat another distance between experimental points but we do not know about it. As a result, it is measured up to momentum $p_{\max }$ equal to 2.1 [a.u.] (in atomic units) instead of 2 [a.u.] (above 4 [a.u.] our model density is equal to zero). Taking the same $p_{\max }$ for all spectra and normalizing them to the same area, we observe a very strong reaction of $c_{m}(\varphi)$ in the case of model shown in Fig. 1 of the paper [1]. Because this model is much more strongly anisotropic than real electronic densities, the same test was performed for projections presented here in Fig. 1. For this model the changes in $c_{m}(\varphi)$ were lower but still much higher than for experimental data where their inconsistency was due only to the statistical noise. Namely, inconsistency of $c_{m}(\varphi)$ for the incorrect model projection was of the order: $2 \%$ for $c_{1}(\varphi), 5 \%$ for $c_{2}(\varphi)$ (condition $1^{\circ}$ ) and from $10 \%$ up to $40 \%$ for $c_{3}(\varphi)$ and $c_{4}(\varphi)$, respectively (condition $2^{\circ}$ ).

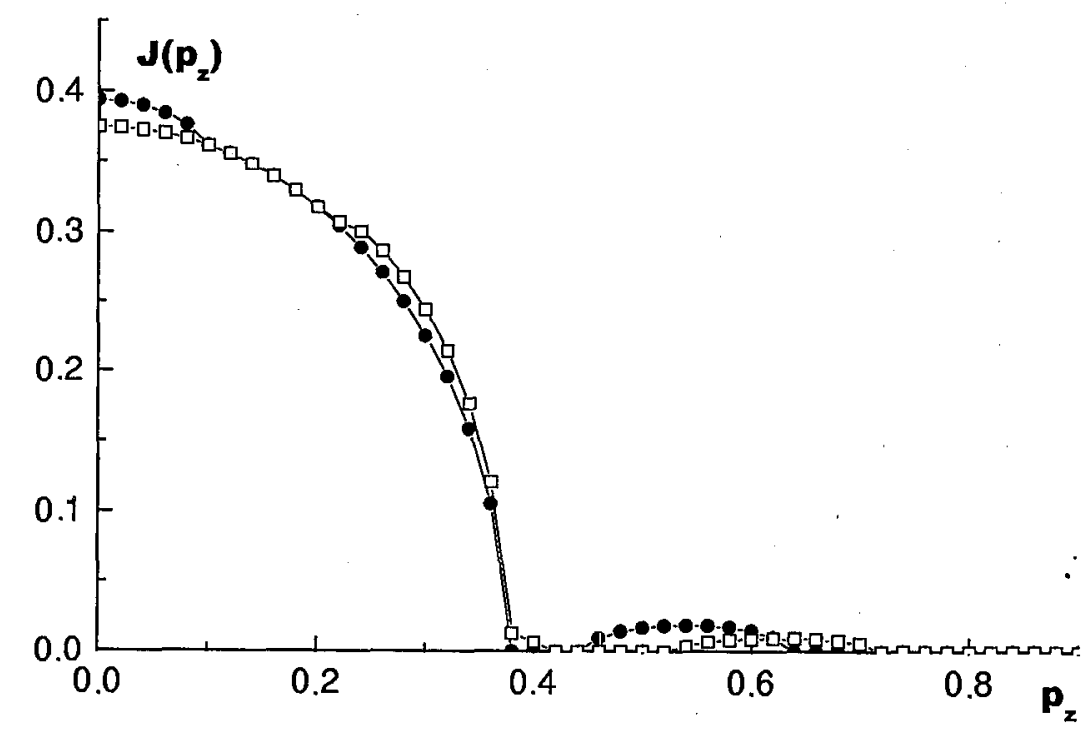

Fig. 1. Model projections $J\left(p_{z}\right)$ for the hcp structure with $p_{z}$ along $\Gamma K$ (circle) and the $\Gamma M$ (square) without an isotropic core contribution (units 2 [a.u.] $\equiv 1$ ). 


\section{Conclusions}

The interdependences between the plane projections of $\rho(p)$, found in Sec. 2, can be utilized for both improvement of the CP (or one-dimensional angular correlation of positron annihilation radiation spectra) and a verification of various techniques used for correcting these experimental data. It could be important particularly for the CP where the way of correcting $J\left(p_{z}\right)$ is not univocal and can be individual for each the spectrum $J\left(p_{z}\right)$ [5].

\section{Acknowledgments}

I am very grateful to Prof. R.N. West for making a vailable his experimental data [5].

\section{References}

[1] G. Kontrym-Sznajd, A. Jura, Acta Phys. Pol. A 95, 586 (1999).

[2] G. Reiter, R. Silver, Phys. Rev. Lett. 54, 1047 (1985); P.E. Mijnarends, Phys. Rev. $160,512(1967)$.

[3] G. Kontrym-Sznajd, M. Samsel, R.N. West, Acta Phys. Pol. A 95, 591 (1999).

[4] R.L. Waspe, R.N. West, in: Positron Annihilation, Eds. P.G. Coleman, S.C. Sharma, L.M. Diana, North-Holland, Amsterdam 1982, p. 328; P.A. Walters, J. Mayers, R.N. West, ibid., p. 334; S.B. Dugdale, H.M. Fretwell, M.A. Alam, G. Kontrym-Sznajd, R.N. West, S. Badrzadeh, Phys. Rev. Lett. 79, 941 (1997).

[5] L. Dobrzyński, private communication. 